\title{
Visual Attention Control for a Legged Mobile Robot based on Information Criterion
}

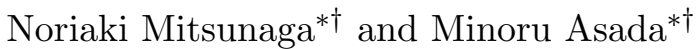 \\ mitchy@er.ams.eng.osaka-u.ac.jpasada@ams.eng.osaka-u.ac.jp \\ ${ }^{*}$ Dept. of Adaptive Machine Systems, $\dagger$ HANDAI Frontier Research Center, \\ Graduate School of Engineering, Osaka University
}

\begin{abstract}
Visual attention is one of the most important issues for a vision guided mobile robot. Methods have been proposed for visual attention control based on information criterion[3, 4]. However, the robot had to stop walking for observation and decision. This paper presents a method which enables observation and decision more efficiently and adaptively while it is walking. The method uses the expected information gain from future observations for attention control and action decision. It also proposes an image compensation method to handle the image changes due to the robot motion. Both are used to estimate observation probabilities from the observation while it is walking and then action probabilities are estimated from a decision tree based on the information criterion. The method is applied to a four legged robot. Discussions on the visual attention control in the method and the future issues are given.
\end{abstract}

\section{Introduction}

Mobile robots are often equipped with visual sensors that bring a huge amount of data about the environment. For efficient decision making, attention control which extracts necessary and sufficient information for the given task is necessary. Gaze control is the one of most important attention control for a robot equipped with a limited view angle camera since the decision making is highly depends on it. We have proposed a method of efficient observation for decision making with gaze control based on information criterion $[3,4]$. However, the methods assumed a stop-observation-act approach. To make decisions much more efficiently, observation while it is taking some action, for example walking, has been desired.

To use observations while it is walking, the following issues arises, 1) there are much more disturbances to such observation than the stop-and-observe one, 2) it may move too much between gaze changes to regard the observation is done at the same location or it must consider where the observation is done, 3 ) it should stop walking or slow down the motion when it cannot make any decisions. Kosaka et al.[2] have proposed retroactive updating of position uncertainty to handle the second problem. Moon et al.[5] have proposed a view point planning considering the second and the third problems for navigation tasks. Burgard et al.[1] proposed a Markov localization method which determines which direction to move for localization. However, their method have not considered the second nor the third problem. Also main purpose of all these methods was for localization and efficiency of observation was considered from a view point of localization only. Further, they assumed the wheeled mobile robots of which observation is rather stable and therefore ignored the first problem.

In this paper, we propose a method of image compensation mechanism for decision making to handle the first and the second problems. Further, we propose to use the expected information gain as a reliability measure for third problem. Action decision is done by a decision tree constructed by information criterion based on the training data.

The rest of the paper is organized as follows. First, the method to construct action decision tree is introduced along with basic ideas related to the information criterion, the efficient observation, and the decision making. Also the expected information gain as a reliability measure is introduced. Then, the compensation method for image changes by walking is proposed, and the experimental results using the RoboCup four-legged robot league platform are shown. Finally, discussion and the future issues are shown.

\section{The method}

\subsection{Assumptions}

In our experiments, the robot has to pan and tilt its camera to acquire the necessary information for action selection since the visual field of the camera 
is limited. The environment includes several landmarks of which appearances provide the robot with sufficient information to uniquely determine the action. Training data are given for making decisions.

We used a teaching method to collect such data. A training datum consists of a set of the views of the landmarks at the current position and the action to accomplish the task. During the training period, the robot pans and tilts its camera head to observe as many landmarks as possible. The robot stops its leg motions while rotating the head to guarantee that the landmarks are observed from the same location. We separately prepare image data for image compensation of locomotion and image shakes by walking.

\subsection{Information gain by observation}

Suppose we have $r$ kinds of actions and $n$ training data. First, the occurrence probabilities of actions $p_{j}$ $(j=1, \ldots, r)$ are calculated from $p_{j}=n_{j} / n$, where $n_{j}$ denotes the number of taken action $j$. Therefore, the entropy $H_{0}$ for the action probability is given by

$$
H_{0}=-\sum_{j=1}^{r} p_{j} \log _{2} p_{j}
$$

Next, the occurrence probabilities of actions after observation are calculated. After the observation, it knows whether the landmark is inside the attention window $\left(\theta_{L k}, \theta_{U k}\right]$ or not. We denote the number of times action $j$ was taken as $n_{i j k}^{I}$ when the landmark $i$ was observed in $\left(\theta_{L k}, \theta_{U k}\right]$ and $n_{i j k}^{O}$ when not observed. Then, the occurrence probability becomes,

$$
p_{i k j}^{I, O}=n_{i k j}^{I, O} / n_{i k}^{I, O},
$$

where $n_{i k}^{I}=\sum_{j}^{r} n_{i k j}^{I}$, and $n_{i k}^{O}=\sum_{j}^{r} n_{i k j}^{O}$. Next, the entropy after the observation are calculated as follows:

$$
H_{i k}=-\sum_{x=\{I, O\}} \frac{n_{i k}^{x}}{n_{i k}} \sum_{j=1}^{r}\left(p_{i k j}^{x} \log _{2} p_{i k j}^{x}\right) .
$$

The information gain by this observation $I_{i k}$ is $H_{0}-H_{i k}$. The larger $I_{i}$ is, the smaller the uncertainty after the observation is.

When the time for observation is constant, we can use the information gain to make action decision tree. However, the time for observation changes depending on the gaze directions. Therefore, we use the information gain per time, in other words the velocity, rather than information gain itself.

We denote $T$ as the time to get the observation after previous one, and the information gain per time $i_{i k}$ is,

$$
i_{i k}=\frac{I_{i k}}{T+T_{C}}=\frac{H_{0}-H_{i k}}{T+T_{C}}
$$

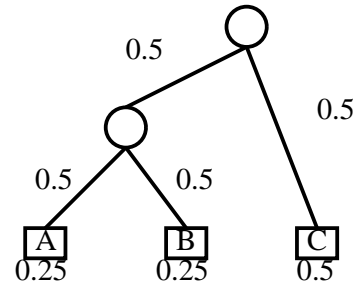

Figure 1: An example action decision tree.

where $T_{C}$ is a positive constant. When the direction is already observed $T=0$. To keep simplicity, we limit pan and tilt angles into several discrete values and the robot select an observing direction nearest to the attention window from them.

\subsection{Making an action decision tree}

To make an action decision tree, 1) we calculate each information gain per time $i_{i k}$ for action after observing a window $k$ of landmark $i$, and 2) divide the data set according to the attention window with the largest information gain per time. We iterate these processes until the information gains for all windows become zero or the action in the subset of the training data becomes unique. In the action decision tree, a node, an arc, and a leaf indicate the window to divide the data set, whether the landmark is observed in the window, and the action to be taken, respectively. As a result attention windows are in decreasing order of uncertainty after its observation.

\subsection{Making a decision}

In order to make an action decision, first, the robot sets the observation probabilities to attention windows of the currently or previously observed directions, and 0.5 to windows of never observed directions. Then it calculates action probabilities using the observation probabilities. An action probability is the sum of the probability to reach leaves to take the action in the tree. If one of the action probabilities is very high, that action would be the correct one. However, in some cases it is dangerous to use action probabilities only. For example, in the action decision tree of Figure 1, the action probability of $\mathrm{C}$ is 0.5 before any observation is done.

Here, we propose to use an expected information gain as a measure of reliability. We define total expected information gain as,

$$
-\sum_{\text {all node }}\left(\frac{n_{\text {node }}}{n}\right) I_{\text {node }}\left\{p \log _{2} p+(1-p) \log _{2}(1-p)\right\}
$$




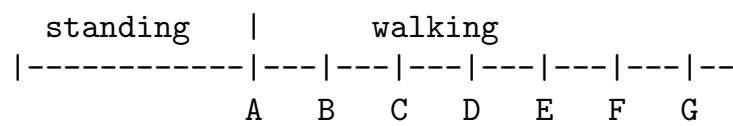

Figure 2: A time sequence example. At time $A$, it starts walking. $A, B, C, \ldots$ are the beginning of a walking period.

where $n_{\text {node }}$ is the number of training data that this node has and $I_{\text {node }}$ is the expected information gain which is calculated in building the tree, and $p$ is the probability that the landmark is in the attention window of the node. Since the entropy of $p$, $p \log _{2} p+(1-p) \log _{2}(1-p)$ ranges from 0 to 1 , and it becomes 1 when the $p=0.5$ or mostly ambiguous, we use this as the reliability measure.

When the total expected information gain is smaller than the threshold and one of the action probability is over the threshold, it takes that action. Then, it observes the direction of the largest expected information gain while it is taking the action. Otherwise, until one of them becomes high enough, it stops walking and continues to check the direction of the largest expected information gain, updates the observation probability, and the action probabilities.

\subsection{Image compensation}

We need some kind of compensation mechanism to make decisions by using the action decision tree built by statically taken images. First, it should compensate for locomotion which is common for both wheeled and legged robots. Second, it should compensate for the shaking motion by its walking, that can be ignored by wheeled and slowly moving legged robots. Compensation of camera images for walking is necessary for dynamic walking robots. While it may possible to compensate the camera movement by active or passive control, high speed feedback or complicated mechanism are needed. Another method is to compensate the image after it has been taken. With this, we do not need precise control to compensate its walking. We take this approach.

To compensate the above two, we calculate the compensation values from images taken by the walking sequences (Figure 2). At first, the robot is standing still, at time $\mathrm{A}$ it starts walking, and time $\mathrm{B}$ is the beginning of the second walking period.

We denote the time of $\mathrm{A}, \mathrm{B}, \mathrm{C}, \ldots$ as $t_{A}, t_{B}, t_{C}, \ldots$, and, the camera image at time $t$ as $U_{t}$. At the beginning of the walking periods, for example, the difference between $U_{t_{A}+i}$ and $U_{t_{B}+i}$ includes the effect of both the shaking due to the beginning of the walking and locomotion. When it walks regularly, for example, the difference between $U_{t_{F}+i}$ and $U_{t_{G}+i}$ only includes the effect of locomotion. Here we define the difference of images by,

$\operatorname{Diff}(i, j, \triangle x, \triangle y)=\sum\left\{u_{i}(x, y)-u_{j}(x-\triangle x, y-\triangle y)\right\}^{2}$,

where $u_{i}(x, y)$ denotes the value of the pixel at the point $(x, y)$ in image $U_{i}$. We calculate and find the $(\triangle x(t), \triangle y(t))^{T}$ which makes the smallest $\operatorname{Diff}(t, \quad t+1, \triangle x, \triangle y)$ for each $t$. $(\triangle x(t), \triangle y(t))^{T}$ is the movement of camera in the image coordinate. The movement of the camera in one walking period is,

$$
\sum_{j=t}^{t+W}(\triangle x(j), \triangle y(j))^{T},
$$

where, $W$ is the walking period and $t$ is the time in the period of regular walking. We calculate the mean $\hat{\boldsymbol{x}}_{L}$ and the variance $\boldsymbol{\sigma}_{L}$ of the movement. The $\hat{\boldsymbol{x}}_{L}$ and $\boldsymbol{\sigma}_{L}$ indicates the mean and the variance of the its locomotion in the camera coordinate.

The difference of the images between in standing still and in walking includes the effect of shaking and locomotion. So the effect of shaking at time $t$ calculated by subtracting the effect of locomotion,

$$
\sum_{j=0}^{t}(\triangle x(j), \triangle y(j))^{T}-\frac{\left(t-t_{k}\right) \hat{\boldsymbol{x}}_{L}}{W},
$$

where $t_{k}$ is the beginning of the walking period which includes $t$. We calculate the mean $\hat{\boldsymbol{x}}_{S}(\theta)$ and the variance $\boldsymbol{\sigma}_{S}(\theta)$ of this effect for each walking phase $\theta$.

We use $\hat{\boldsymbol{x}}_{L}, \boldsymbol{\sigma}_{L}, \hat{\boldsymbol{x}}_{S}(\theta)$, and $\boldsymbol{\sigma}_{S}(\theta)$ for the calculation of observation probabilities. When a landmark $i$ is observed in $\boldsymbol{x}$ at time $t_{1}$, the mean and variance of the landmark location in image at time $t$ is,

$$
\begin{aligned}
& \overline{\boldsymbol{x}}(t)=\boldsymbol{x}+\hat{\boldsymbol{x}}_{S}\left(\theta_{t_{1}}\right)+\frac{\left(t-t_{1}\right) \hat{\boldsymbol{x}}_{L}}{W}, \\
& \overline{\boldsymbol{\sigma}}(t)=\boldsymbol{\sigma}_{S}\left(\theta_{t_{1}}\right)+\frac{\left(t-t_{1}\right) \boldsymbol{\sigma}_{L}}{W},
\end{aligned}
$$

where $\theta_{t_{1}}$ is the walking phase at $t_{1}$. We use the ratio of the area made by $\bar{x}(t)-\bar{\sigma}(t)$ and $\bar{x}(t)+\bar{\sigma}(t)$ and the area which is overlapped by the attention window on this area as the observation probability.

\section{Experiments}

\subsection{Task and Environment}

We used a legged robot with a limited view angle for the RoboCup SONY legged robot league (Figure 3 ). We used the half of the field, there are 4 landmark poles, a goal, and a ball. All the landmarks and the ball are distinguished by their colors. The task is to 


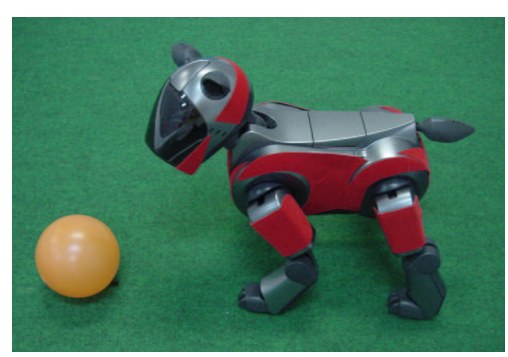

Figure 3: The SONY legged robot for the RoboCup SONY legged robot league.

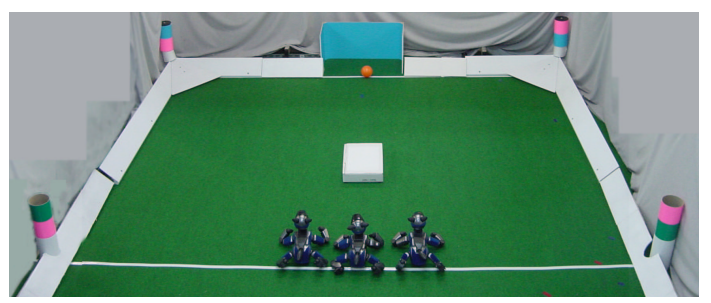

Figure 4: Experimental field (same as the one for the RoboCup SONY legged robot league).

move to the position where the ball and the goal are in line based on the visual information. The robot must avoid obstacle which is not distinguishable by its color.

The view angle (number of image pixels) of the robot's camera are about 58 degrees (88 pixels) in width, and about 48 degrees (72 pixels) in height. Each leg and the neck have three degrees of freedom. The robot can rotate the pan joint from -88 to 88 degrees and the tilt joint from -80 to 43 degrees. The maximum frame rate of the camera is $40[\mathrm{~ms}]$. We prepared three actions, move forward, left forward, right forward. These actions are based on trot gait and were developed without consideration for image shaking.

As vision sensors, we used the coordinates of the image centers of the landmarks and the ball, the minimum and maximum $\mathrm{x}, \mathrm{y}$ coordinates (totally four) of the goals. We used a pair of the pan $(x)$ and the tilt $(y)$ angles as a sensor value, or we divided training data set by the observation to check whether a sensor value is in the rectangle attention window $\left(x_{\min }, y_{\min }\right)-\left(x_{\max }, y_{\max }\right)$ or not.

\subsection{Image compensation}

Figure 5 shows the images while the robot is watching the front direction and doing forward motion. The images are taken every $80[\mathrm{~ms}]$ and the walking period is $600[\mathrm{~ms}]$. Figure 6 shows the $\Delta y$ of three trials of forward motions. We can see high jumps in

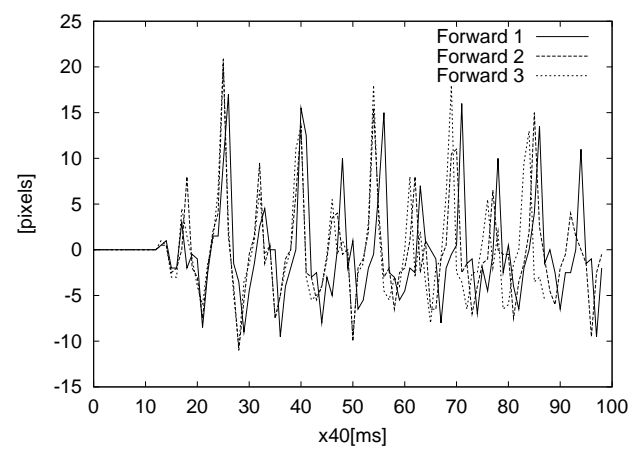

Figure 6: The $\triangle y$ of forward motion.

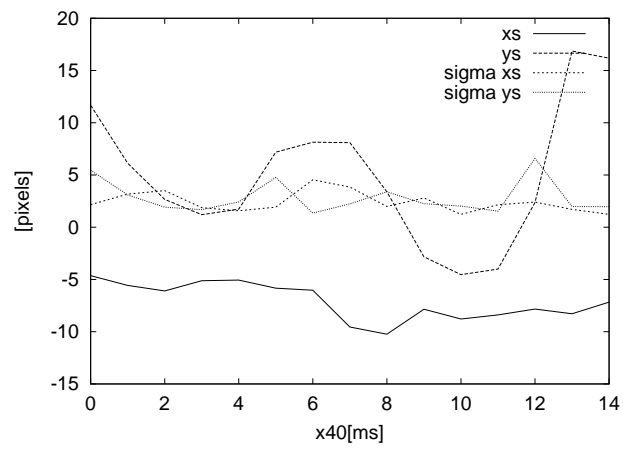

Figure 7: The $\hat{\boldsymbol{x}}_{S}$, and $\boldsymbol{\sigma}_{S}$ of the forward motion watching at front direction.

every walking periods. Figures 7,8 , and 9 show the $\hat{\boldsymbol{x}}_{S}$, and $\boldsymbol{\sigma}_{S}$ of the forward, right forward, and left forward actions watching at front direction.

\subsection{Experimental results}

We trained the robot starting from one of three positions in the middle of the field. We prepared five directions (every 44 degrees) in the pan joint and five directions (every 21 degrees) in the tilt joint to observe. In order to guarantee that it can observe same view angles by observing one direction in spite of the shaking motions, we narrowed the angles than camera's field of view.

We obtained 239 training data and constructed a action decision tree. Figure 10 shows the attention windows generated with proposed method. Figures 11,12 , and 13 show the changes of expected information gain and the maximum action probability when the robot took actions beginning from the center of the field based on the constructed action decision tree.

Figure 11 shows the result when action probability threshold was 0.4 and the information gain threshold 

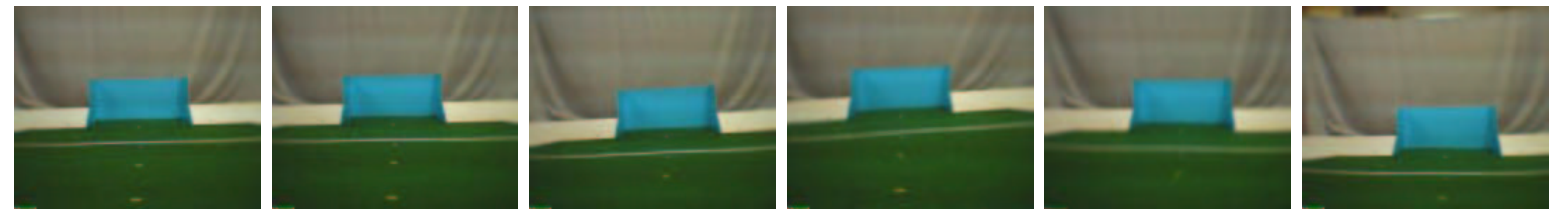

Figure 5: The images of the camera while the robot is moving forward. The images are taken every 80[ms].

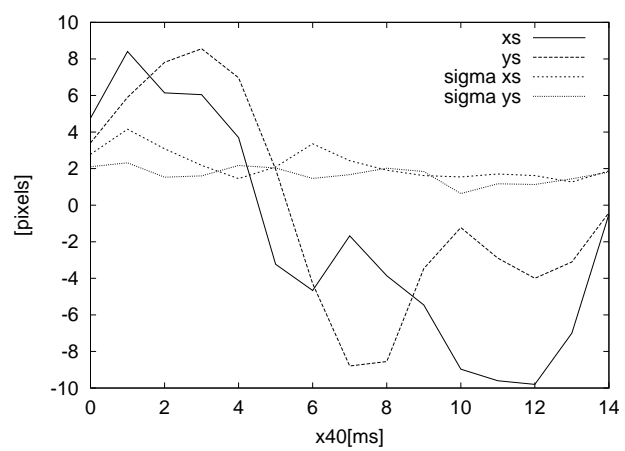

Figure 8: The $\hat{\boldsymbol{x}}_{S}$, and $\boldsymbol{\sigma}_{S}$ of the right forward motion watching at front direction.

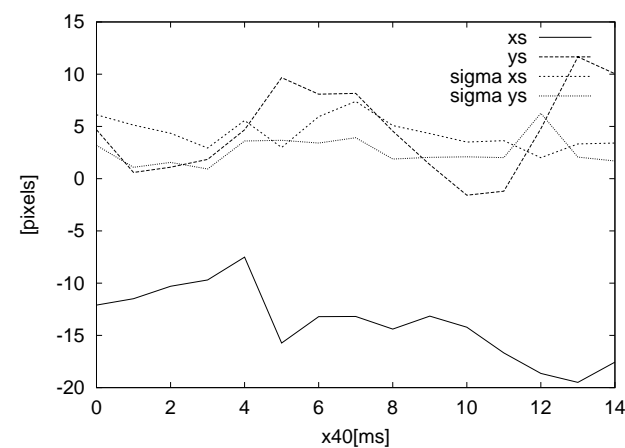

Figure 9: The $\hat{\boldsymbol{x}}_{S}$, and $\boldsymbol{\sigma}_{S}$ of the left forward motion watching at front direction.

was not used. The time the robot reached to the goal position was shorter and the frequency of standing up for observation was lower. However the robot ignored the obstacle so the task has not been completed.

Figure 12 shows the result when action probability threshold was 0.9 and the information gain threshold was not used. We tried with the threshold 0.7 and 0.8 and it tried to avoid the obstacle but it hit to the obstacle. With the threshold 0.9 it avoided the obstacle and reached to the goal position. However, it frequently stand up for observation.

Figure 13 shows the result when action probability

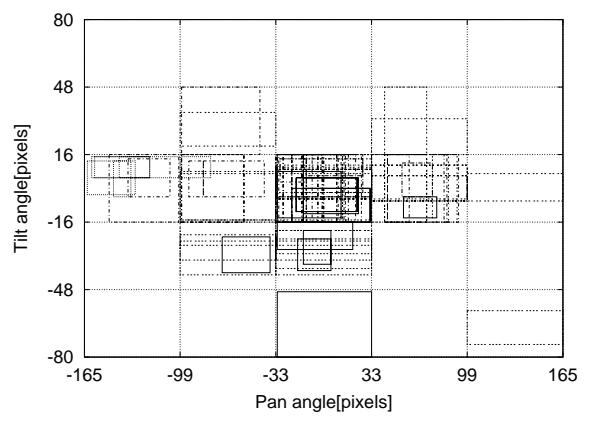

Figure 10: Created attention windows by proposed method.

threshold was 0.4 and the information gain threshold was 0.3 . It shows that by using the information gain threshold, the frequency of standing up for observation is higher than without the threshold and lower than high action probability threshold. And the robot reached to the goal position avoiding the obstacle. This shows the validity of the information gain threshold.

\section{Discussions and conclusions}

Currently, training data should cover the case of sensor noises or of occlusions in order to handle these cases, then we may need large training data. A method which can find robustness of observation may be desired. We have to determine the $T_{C}$ for calculation of information gain per time, and two thresholds for maximum action probability and expected information gain for each task. These parameter should be studied.

We proposed a visual attention control for a legged mobile robot. It consisted of a decision tree constructed by information gain by time and the compensation mechanism for walking and locomotion. We introduced the expected information gain as a measure of reliability of decision. Attention control is done by observing the direction which has largest expected information gain calculated with the decision tree. The validity of the method was shown with reaching task by a four legged robot. 


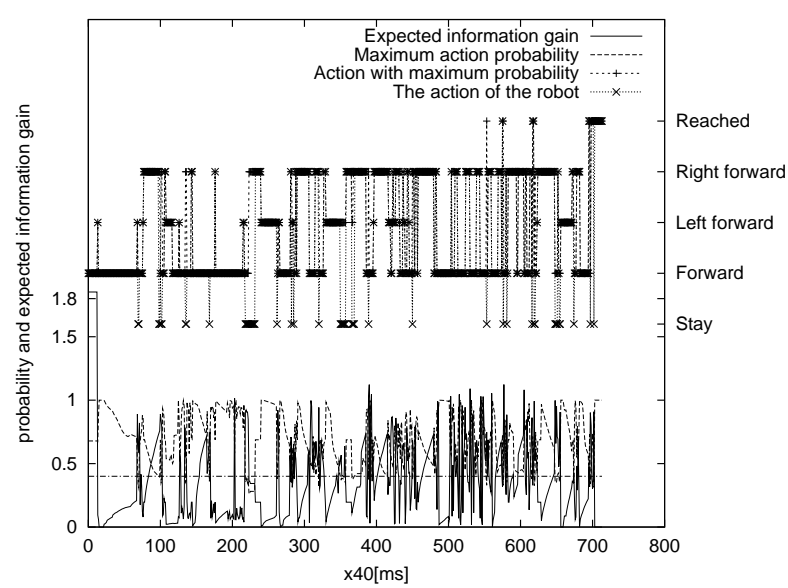

Figure 11: Changes of expected information gain, the maximum action probability, actions which had maximum action probabilities, and which was taken by the robot. The starting point of robot was the center of the field. The action probability threshold was 0.4 and the information gain threshold was not used.

\section{Acknowledgments}

This research was supported by the Japan Science and Technology Corporation, in Research for the the Core Research for the Evolutional Science and Technology Program (CREST) titled Robot Brain Project in the research area "Creating a brain."

\section{References}

[1] W. Burgard, D. Fox, and S. Thrun. Active mobile robot localization. In Proceedings of the Fourteenth International Joint Conference on Artificial Intelligence (IJCAI). Morgan Kaufmann, San Mateo, CA, 1997.

[2] A. Kosaka, M. Meng, and A. C. Kak. Vision-guided mobile robot navigation using retroactive updating of position uncertainty. In Proceedings of the 1993 IEEE International Conference on Robotics and Automation, volume 2, pages 1-7, 1993.

[3] N. Mitsunaga and M. Asada. Observation strategy for decision making based on information criterion. In Proceedings of the 2000 IEEE/RSJ International Conference on Intelligent Robots and Systems, pages 10381043. 2000.

[4] N. Mitsunaga and M. Asada. Sensor space segmentation for visual attention control of a mobile robot based on information criterion. In Proceedings of the 2001 IEEE/RSJ International Conference on Intelligent Robots and Systems, pages 1714-1719. 2001.

[5] I. Moon, J. Miura, and Y. Shirai. Dynamic motion planning for efficient visual navigation under uncertainty. In Y. Kakazu, M. Wada, and T. Sato, editors, In Proc. of the Intelligent Autonomous Systems 5, pages 172-179, 1998.

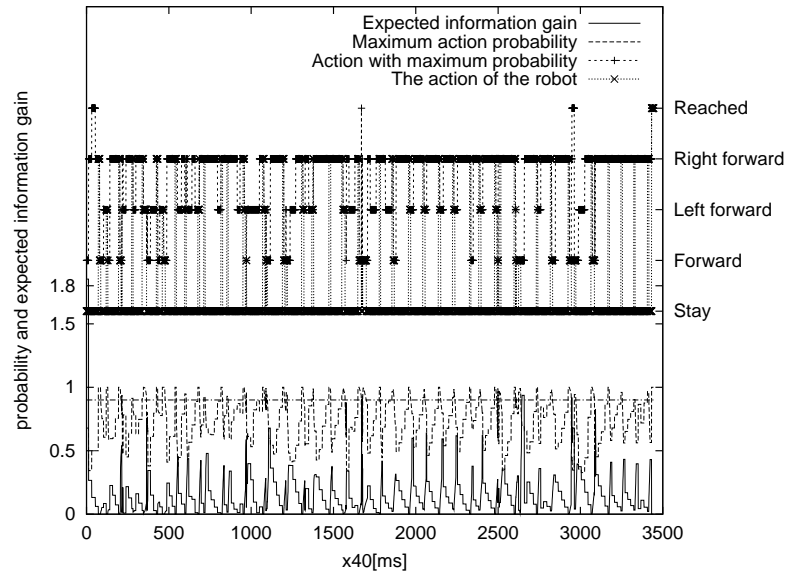

Figure 12: Changes when the starting point of robot was the center of the field. The action probability threshold was 0.9 and the information gain threshold was not used.

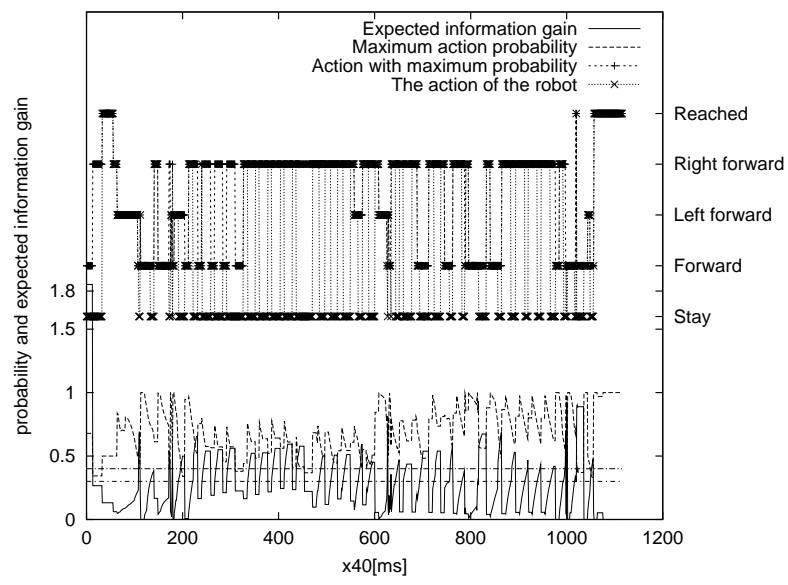

Figure 13: Changes when the starting point of robot was the center of the field. The action probability threshold was 0.4 and the information gain threshold was 0.3 . 